\begin{abstract}
DER BERICHT HERODOTS über "den häßlichsten Brauch der Babylonier" ( $\delta$...

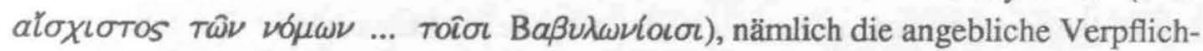
tung einer jeden Frau des Landes (

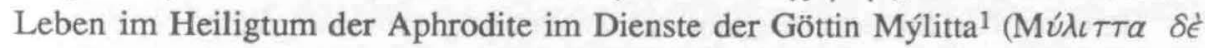

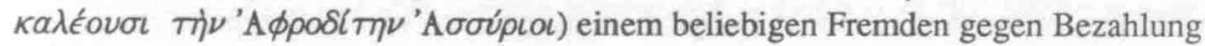
hinzugeben, hat eine außerordentlich verzweigte Rezeptionsgeschichte, die bisher erst ansatzweise dargestellt worden ist. Früh ein Gegenstand der Herodot-Kritik, hat die Erzählung mit der zunehmenden Verselbständigung der Einzelwissenschaften mehrere Hypostasen angenommen, die seit langem ihr Eigenleben führen. Die Pole zwischen denen sich dieses Eigenleben abspielt, sind die Anerkennung der Faktizität des angeblichen babylonischen Brauchs einerseits und die Zurückweisung nicht nur des Herodoteischen Berichts, sondern jeglicher Form von Tempelprostitution für den Bereich der keilschriftlichen altorientalischen Quellen andererseits.

Angesichts des fast völligen Fehlens von Vorarbeiten kann es nicht die Absicht dieses Aufsatzes sein, die Rezeptionsgeschichte von Klio 199 mit dem Anspruch
\end{abstract}

${ }^{1}$ Zum Namen Mýlitta kennt die Herodot-Philologie zwei alte Erklärungstraditionen. Die eine stellt den Namen mit syrisch moladta $\bar{a}$ zusammen und verbindet dies mit dem Epitheton der Venus genitrix; cf. F. Münter, Die Religion der Babylonier, Kopenhagen 1827, 22 ("MúdıtTa, ohne Zweifel

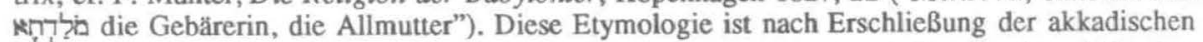
Quellen modifiziert beibehalten worden in der Ableitung von akkad. mu'allittu "Gebärerin"; cf. E. Schrader, Die Keilinschriften und das Alte Testament, Berlin 1903³, 423, 7; E. Meyer, Geschichte des Altertums I/ $2^{3}$ Stuttgart/Berlin 1913, 460; P. Jensen, Die Kosmologie der Babylonier, Strassburg 1890, 515; W. Baumgartner, ArOr 18/1-2, 1950, 82 n. 72; W. Kornfeld, DBS 8, 1972, 1360. $m u^{2}$ allittu ist als Epitheton der Tiamat belegt, kommt aber für die Göttin Istar, die gerade keine Muttergöttin ist, nicht in Frage. Die andere, ebenfalls bis heute vertretene Etymologie leitet Mýlitta von semit. ba ll "Herrin" ab; sie findet sich bei F. Creuzer, Herodoti Musae I, London 1830, 446 Anm.; H. Stein, Herodotos, London 19016 226 n. 16; E. Meyer, Geschichte des Altertums I, Stuttgart 1884, 177, und sonst. Der erste assyriologische Beitrag zu der Frage kann heute der Kritik nicht mehr standhalten: H. C. Rawlinson bezieht sich in dem unten n. 29 zitierten Werk p. 595 auf

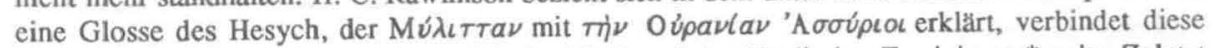
Nachricht mit sumerisch $\mathrm{m} 1$ "Stern" und schließt auf ein akkadisches Femininum *multa. Zuletzt hat S. Dalley, RA 73, 1979, 177 sq., den Namen Múdıtra als Wiedergabe der nA und spB Aussprache des Namens der Göttin Ninlil (mul(l)is(s)u, mullēsu, ${ }^{*}$ mullišsu) gedeutet. Cf. dazu mit

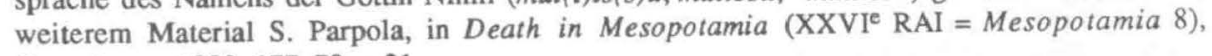
Copenhagen 1980, 177-78 n. 21. 
auch nur auf eine annähernde Vollständigkeit der Belege zu schreiben. Es sollen vielmehr nur einige ihrer Hauptlinien, auch unter Benutzung teilweise zufälliger Lesefrüchte, skizziert werden. Darüberhinaus soll die Frage der Tempelprostitution im Alten Orient anhand erst unlängst erschlossener Quellen erneut aufgeworfen und schließlich soll dem immer noch sehr schemenhaften Bild ein weiterer, bisher unbekannter Mosaikstein hinzugefügt werden, der überraschenderweise aus den Schatzkammern der Nuzi Collection des Harvard Semitic Museum zutage kam, die der Obhut des Jubilars in seiner Eigenschaft als Kurator dieser Institution anvertraut sind. Dem Dank für die Überlassung der Publikationsgenehmigung sei der Dank für die von Anfang an gastliche und rasch freundschaftliche Aufnahme in Cambridge und Belmont hinzugefügt.

Herodots Bericht über babylonische Sitten und Gebräuche, die er bei seinem wohl nur kurzen Aufenthalt ${ }^{2}$ in Babylon wahrscheinlich bereits in typisch dragomanischer Anekdotik aus dem Munde seiner einheimischen Gewährsmänner kennenlernte, ist bei den antiken Schriftstellern offenbar nicht expressis verbis erörtert worden. Die angebliche Sitte der einmaligen Prostitution aller Babylonierinnen ist aber ohne Hinweis auf Herodot, doch vielleicht in Abhängigkeit von ihm, gelegentlich erwähnt, nämlich bei dem wohl im 2. Jahrhundert v. Chr. lebenden Verfasser des apokryphen Briefes des Jeremia ${ }^{3}$ und bei Strabo. ${ }^{4}$ Die Grundhaltung der Antike Herodot gegenüber war im wesentlichen negativ. Mehrere antike Autoren haben, nicht zuletzt um die Zuverlässigkeit ihrer eigenen Geschichtswerke zu betonen, Herodot kritisiert; dies ist von Ktesias ${ }^{5}$ und Manetho ${ }^{6}$ bekannt. Ein wohl im 2. Jhdt. n.Chr. lebender Aelios Harpokration hat über die falschen Behauptungen Herodots ein Buch verfaßt. ${ }^{7}$ Josephus kommt zu dem Urteil, daß alle griechischen Autoren die Wahrheitswidrigkeit des Werkes Herodots zugäben, ${ }^{8}$ Plutarch spricht gar von seiner kakoetheia. ${ }^{9}$ Selbst ohne ausdrückliche Erwähnung des Berichts über Babylon sind gewiß auch auf ihn manche Verdammungsurteile zu übertragen wie das Diodors über das, "was nun Herodotos und einige Andere, welche über Ägyptische Geschichte geschrieben, gefaselt haben, indem sie anstatt der Wahrheit lieber wunderbare

${ }^{2}$ Cf. F. Jacoby, Herodot, in RE Suppl. 2, 205-520.

3"Brief des Jeremia" (= Baruch VI) 44.

${ }^{4}$ Strabo XVI 1.20; cf. Strabo vol. VII (The Loeb Classical Library), London/Cambridge, Mass. $1966^{4}, 227$.

${ }^{5}$ Cf. F. Jacoby, RE XI, 2, 2041 sqq.

6Josephus, Contra Apionem I 73.

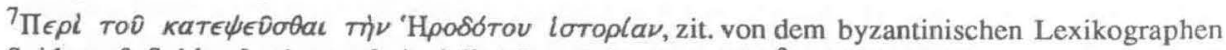
Suidas; cf. Suidae Lexicon, ed. A. Adler, Pars I, Stuttgart 1971², 367 Nr. 4013.

${ }^{8}$ Contra Apionem I 3.

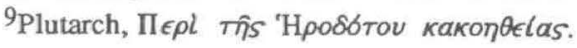


Erzählungen vorführen und Sagen erfinden wollten, um ihre Leser zu reizen", ${ }^{10}$ oder das Lukians, der Herodot zusammen mit Ktesias und darüberhinaus allen Dichtern unter Einschluß Homers vorwarf, daß sie "ihre Lügen sogar aufgeschrieben und also nicht nur ihre gleichzeitigen Zuhörer damit betrogen, sondern sie durch den Reiz ihres schönen Stils und die Musik ihrer Verse bis auf uns fortgepflanzt haben". ${ }^{11}$ Die positiven Urteile, die diesen Äußerungen gegenüberstehen, beziehen sich nicht auf den Wahrheitsgehalt, sondern den schönen Stil der Herodoteischen Historien. ${ }^{12}$

Die Wiederentdeckung Herodots im 15. Jahrhundert ${ }^{13}$ war zunächst von den negativen Urteilen der Antike stark beeinflußt, bis sich im 16. Jahrhundert eine Änderung der Einstellung abzeichnete, die in Verteidigungen Herodots ihren Ausdruck fand ${ }^{14}$ und die auf die unmittelbare Konfrontation der Epoche mit bis dahin unbekannten Kulturen, fremdartigen Sitten und Gebräuchen und auf die damit verbundene erhöhte Wertschätzung von Reiseberichten und Antiquaria zurückgeführt worden ist. ${ }^{15}$

Die Frage nach der Glaubwürdigkeit Herodots und gerade auch die Bewertung der Beschreibung der babylonischen Zustände hat im 18. Jahrhundert eine über den Gegenstand als solchen weit hinausgehende grundsätzliche Bedeutung gewonnen. Voltaire, der mit seinen großen Geschichtsdarstellungen ${ }^{16}$ sich direkt der Problematik historischer Quellen gegenübersah und "wie viele seiner Zeitgenossen ... vom 'Pyrrhonisme de l'histoire' (der historischen Skepsis)"17 beeinflußt war, hat in seiner "Philosophie de l'histoire", die später seinem großen Geschichtswerk "Essay sur les mœurs et l'esprit des nations" als Einleitung vorangestellt wurde, gerade Herodots Bericht über die allgemeine Prostitutionspflicht in Babylon als Beispiel einer Quelle

${ }^{10}$ Diodor I 69; Übersetzung von A. Wahrmund, Diodor's von Sicilien Geschichts-Bibliothek I, Stuttgart 1866, 114.

${ }^{11}$ Lukian, Philopseudes 2; Übersetzung von Ch. M. Wieland (Lukian, Werke in drei Bänden, Berlin/Weimar 1974, I 86).

${ }^{12}$ Zur Herodot-Rezeption in der Antike cf. K.-A. Riemann, "Das Herodoteische Geschichtswerk in der Antike," Diss. München 1967.

${ }^{13}$ Als Kuriosum sei vermerkt, daß mehrere Handschriften des 15. Jhdts. gerade Klio 199 auslassen; cf. A. D. Godley, Herodotos I (Loeb Classical Library), London/Cambridge 1966 6 , 250 n. 1.

${ }^{14}$ Cf. z.B. Henricus Stephanus, Apologia pro Herodoto, 1566.

${ }^{15}$ A. Momigliano, "The place of Herodotus in the history of historiography," History 43, 1958, 113.

${ }^{16}$ Insbesondere "Le siècle de Louis XIV" und "Histoire de Charles XII, Roi de Suède". Ein besonderes Interesse Voltaires an der vor allem durch Herodot vermittelten altorientalischen Geschichte
wird verschiedentlich, insbesondere aber in einigen seiner Tragödien ("Sémiramis", "Les Scythes", "La princesse de Babylone") deutlich.

17J. H. Brumfitt, "Die Geschichtsphilosophie Voltaires," in Voltaire, ed. H. Baader (Wege der Forschung 286), Darmstadt 1980, 91 (deutsche Öbersetzung von Voltaire Historian, Oxford 1958, Kap. V: The Philosophy of History). 
ausgewählt, die einer mit den Normen der menschlichen Natur messenden Kritik nicht standhält. Verdienst und Grenzen des Geschichtsverständnisses der Aufklärung werden hier paradigmatisch deutlich, indem die Kritik gegenüber der unbedingten Autorität der Tradition einer wissenschaftlichen Geschichtsforschung den Weg bereitet, die Norm dieser Kritik aber wesentlich unhistorisch ist und so die Gefahr birgt, Phänomene vergangener und fremder Gesellschaften zu negieren, die sich aus der als menschliche Natur definierten Rationalität der Aufklärung nicht ableiten lassen. Voltaire schreibt über den von Herodot behaupteten babylonischen Brauch:

De bonne foi, cette infamie peut-elle être dans le caractère d'un peuple policé? Estil possible que les magistrats d'une des plus grandes villes du monde aient établi une telle police? que les maris aient consenti de prostituer leurs femmes? que tous les pères aient abandonné leurs filles aux palefreniers de l'Asie? Ce qui n'est pas dans la nature n'est jamais vrai. ${ }^{18}$

Die Kontroverse, die sich hieran anschloß, ist unlängst von D. Arnaud in einem Vortrag, ${ }^{19}$ auf den wir im folgenden noch zurückkommen werden, ausgegraben und ins Museum der altorientalistischen Vorgeschichte eingebracht worden. Voltaires Entgegnung auf seinen Kritiker wiederholt das Argument, der Bericht Herodots widerspreche der Natur des Menschen:

Peut-on croire que dans Babylone, dans la ville la mieux policée de l'Orient, des hommes si jaloux de leurs femmes les aient envoyées toutes se prostituer dans un temple aux plus vils étrangers? que tous les époux et tous les pères aient étouffé ainsi l'honneur et la jalousie? que toutes les femmes et toutes les filles aient foulé aux pieds la pudeur si naturelle à leur sexe? Le feseur de contes Hérodote a pu amuser les Grecs de cette extravagance, mais nul homme seusé n'a dû le croire. ${ }^{20}$

Nur am Rande sei vermerkt, daß sich die Geschichtsphilosophie in Deutschland des Themas nur nebenbei und im wesentlichen unkritisch angenommen hat, so Hegel, der sich in seine "Vorlesungen über die Philosophie der Geschichte" fragt, wie die Verpflichtung der Babylonierin zur Prostitution im Tempel der Mýlitta "mit den Religionsbegriffen zusammengehängt habe", 21 und Schopenhauer, der den als Tatsache akzeptierten Brauch als ein extremes Beispiel neben anderen für die Relativität der Sexualehre anführt. ${ }^{22}$

${ }^{18}$ Voltaire, "La philosophie de l'histoire," in CEuvres complètes 22, Aux Deux-Ponts (= Zweibrücken) $1792,56 \mathrm{sq}$.

${ }^{19}$ D. Arnaud, "La prostitution sacrée en Mésopotamie, un mythe historiographique?," RHR 183, 1973, 111-15.

${ }^{20}$ Voltaire, La défense de mon oncle, chap. 2: L'apologie des dames de Babylone, in CEuvres complètes 36, Aux Deux-Ponts 1792, 202-7. Cf. noch Voltaire, Questions sur L'encyclopédie, Art. "Babel"; P.-H. Larcher, Histoire d' Hérodote, Tome I, Paris 1802², 522-27.

${ }^{21}$ G. W. F. Hegel, Sämtliche Werke 11, Stuttgart 19493 247.

${ }^{22}$ A. Schopenhauer, Parerga und Paralipomena, Sämtliche Werke 4, München 1913, 407. 
Die Popularität, die das Thema im 18. Jahrhundert im Anschluß an Voltaire genoß, hat sich auch in der schönen Literatur der Zeit niedergeschlagen. In einem 1798 veröffentlichten, heute wenig bekannten Reiseroman mit dem Titel "Voyages d'Antenor en Grèce et en Asie"23 wird ein Aufenthalt des fiktiven Helden in Babylon geschildert, der sich ganz an Herodot orientiert. Längere Herodot-Zitate, als solche nicht gekennzeichnet, sind in den Text eingefügt. Das rokokoesque Interesse an außereuropäischen Kulturen, die klassizistische Begeisterung für die griechische Antike, der zeitlose Reiz von Kuriosa und Erotica sind die Katalysatoren für einen Trivialroman, der in mancher Hinsicht exemplarisch für den Zeitgeist der letzten Jahrzehnte des 18. Jahrhunderts sein dürfte.

On nous apprit que la fête de Milyta, ou de Vénus, devoit bientôt se célébrer. Je demandai à Azéma quelques détails sur cette fête. "Elle est célébrée, nous dit-elle, dans un temple nommé Socoth-Bonoth. On n'y immole point de victimes; le sang ne coule jamais sur l'autel: la déesse n'y respire que l'odeur de l'encens et des parfums; elle est représentée sur un char conduit par les Amours, et tiré par des cignes ou des colombes".

Arsame alors nous conta la manière dont se fesoient les mariages avant l'établissement de la fête de Milyta. On assembloit toutes les vierges dans un lieu public, les amateurs ou épouseurs les mettoient aux enchères. Il leur étoit permis de les examiner avec la plus scrupuleuse exactitude, et le crieur préposé les adjugeoit au plus offrant. Les plus belles passoient les premières, ensuite les autres, selon les degrés de leur beauté.- - "Cet usage, lui dis-je, étois fort avantageux aux jolies personnes; mais, que fesiez-vous des laides? les envoyiez-vous dans quelque île déserte?-Nous n'étions pas si barbares: on les marioit aussi.-Vous trouviez des acheteurs? -Non; mais on leur donnoit une dot de l'argent qu'on retiroit de la vente des belles, et le peuple, ou les gens peu aisés les épousoient pour leur argent. Depuis l'institution de la fête de Milyta, cette coutume est abolie; mais on a imposé une nouvelle espèce de tribut sur les femmes. Elles sont obligées d'aller une fois dans leur vie au temple de Milyta, pour s'abandonner aux étrangers; elles ne peuvent sans crime refuser leurs faveurs et l'argent qu'on leur offre, quelque modique que soit la somme, car elle appartient à la déesse. Après cette prostitution religieuse, elles sont obligées de passer le rest de leurs jours dans la plus scrupuleuse chasteté.- "Cette dernière loi, dit Phanor, gâte tout, et me paroît ce qu'il y a de plus difficile à observer dans ce dévouement pieux". 24

Nous trouvâmes l'enceinte du temple remplie de femmes charmantes: les plus distinguées, qui ne vouloient point se livrer au premier venu, superbement parées, entourées de leurs domestiques, se tenoient dans leur char sous des voûtes; les autres, ayant une couronne de ficelles autour de la tête, étoient assises sur une pièce de terre. Les unes arrivoient, les autres partoient. Il y avoit des allées, séparées par des cordes tendues, où les étrangers vont choisir la beauté qui leur plait. ...

Je parcourois, avec Phanor et Arsame, ces allées où sembloient respirer l'amour et la volupté. Phanor étoit dans une extase délicieuse: ses yeux s'égaroient, er-

${ }^{23}$ E.-F. Lantier, Voyages d'Antenor en Grèce et en Asie I-III, Paris Année VI [= 1798]. Der Autor (1734-1826), ursprünglich Offizier, orientierte sich sehr stark an dem zehn Jahre zuvor erschienenen vielgelesenen Roman von J.-J. Barthélemy (1716-95), "Voyage du jeune Anacharsis en Grèce."

${ }^{24}$ Lantier, op.cit. Bd. 3, 13 sq. 
roient de l'une à l'autre; il trouvoit toutes les femmes charmantes, toutes allumoient ses desirs. Dans cet enchantement, irrésolu dans son choix, il auroit voulu cueillir toutes les fleurs de ce riant parterre. ...25

Der von Herodot in Klio 199 vorgegebene Stoff scheint sich trotz seiner in Lantiers Roman zum Ausdruck kommenden Anziehungskraft für die männliche Phantasie sonst keiner größeren Wirkung in der Literatur erfreut zu haben. Allerdings gibt es aus jüngster Zeit eine bemerkenswerte Modulation des Themas in dem 1983 erschienenen Roman "Kassandra" der deutschen Schriftstellerin Christa Wolf. Hier wird die kultische Pflicht zur Prostitution aus der Perspektive der Abscheu und Erniedrigung empfindenden Frau erfahren. In Abweichung von Herodot verlegt Christa Wolf die Szene in den Tempelbezirk der Athena von Ilion und verbindet die Prostitutionspflicht mit der Defloration, die von Herodot in diesem Zusammenhang weder erwähnt noch vorausgesetzt wird.

Die Augen schließen, ich kann es nicht mehr, konnte es aber. Öffnete sie einen Spaltbreit und nahm die Beine der Männer in mich auf. Dutzende von Männerbeinen in Sandalen, man sollte nicht glauben, wie verschieden, alle widerlich. An einem Tag kriegte ich fürs Leben genug von Männerbeinen, keiner ahnte es. Ich spürte ihre Blicke im Gesicht, auf der Brust. Nicht einmal sah ich mich nach den anderen Mădchen um, die nicht nach mir. Wir hatten nichts miteinander zu tun, die Männer hatten uns auszusuchen und zu entjungfern. Ich hörte lange, eh ich einschlief, das Fingerschnipsen und, in wieviel verschiedenen Betonungen, das eine Wort: Komm. Um mich wurde es leer, nach und nach waren die anderen Mädchen abgeholt worden, die Töchter der Offiziere, Palastschreiber, Töpfer, Handwerker, Wagenlenker und Pächter. Die Leere kannte ich von klein auf. Ich erfuhr zwei Arten der Scham: die, gewählt zu werden, und die, sitzenzubleiben. Ja, ich würde Priesterin werden, um jeden Preis. ${ }^{26}$

In einer autobiographischen Vortragsreihe zur Entstehung ihrer Erzählung27 zitiert Christa Wolf ausdrücklich Herodot I 199 als ihre Quelle. ${ }^{28}$

Mit dem raschen Aufblühen der assyriologischen Forschung in der Mitte des 19. Jahrhunderts ergab sich fast zwangsläufig die Forderung nach einer Überprüfung Herodots an den neuerschlossenen authentischen Quellen aus dem Zweistromlande selbst. Der erste größere Versuch, die Ergebnisse der noch in der Entzifferungsphase befindlichen Keilschriftkunde in die Herodot-Forschung einzubringen, war die 1858 erschienene Herodot-Ausgabe von George Rawlinson, die zahlreiche Anmerkungen und mehrere Essays des Entzifferers der babylonischen Keilschrift, Sir Henry

${ }^{25}$ Lantier, op.cit. Bd. 3, 33 sq.

${ }^{26} \mathrm{Ch}$. Wolf, Kassandra, Darmstadt/Neuwied 1983, 20.

${ }^{27} \mathrm{Ch}$. Wolf, Voraussetzungen einer Erzählung: Kassandra, Darmstadt/Neuwied 1983.

${ }^{28}$ Nach der Übersetzung von Theodor Braun (Herodot, das Geschichtswerk des Herodotos von Hali-

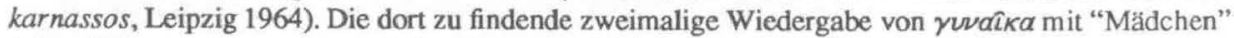
(einmal aber auch rvvaîkes "Weiber") mag zur Einführung des Deflorationsmotivs angeregt haben. 
Creswick Rawlinsons, enthält. ${ }^{29}$ Gilt G. Rawlinson dem abwägenden Urteil F. $\mathrm{Jacobys}^{30}$ als "extrem gläubig" gegenüber den Angaben des pater historiae, so ist das genaue Gegenteil der Fall bei der dreißig Jahre später erschienenen annotierten Edition der ersten drei Bücher der Historien durch A. H. Sayce, ${ }^{31}$ der in seinem Urteil den antiken Kritikern Herodots nicht nachsteht und gar nachzuweisen versucht, Herodot sei nie in Babylon gewesen. Er akzeptiert aber Klio 199 als wahrheitsgemäßen Bericht und verbindet ihn—-soweit ich sehe - erstmals mit der Existenz von Tempelprostituierten, für die er ausdrücklich die qadšātu der assyrischen Texte erwähnt. ${ }^{32}$

In der Folgezeit wurde die Interpretation von Sayce dahingehend modifiziert, daß der Herodoteische Bericht über die Prostitutionspflicht einer jeden babylonischen Frau auf ein Mißverständnis des Brauchs der Tempelprostitution zurückgehe, die nur von bestimmten dafür zuständigen Frauen ausgeübt worden sei. F. Delitzsch schränkt auch diese Aussage auf den "Lokalkultus der Priesterinnen am Anu- und Istar-Tempel zu Erech, dem Tempel der Liebesgöttin" ein, mit dem "die babylonische Ehefrau als solche" "nicht das mindeste zu tun" habe und der auch nicht an jedem babylonischen Tempel existiert habe. ${ }^{33}$ Diese Auffassung ist in der großen Kulturgeschichte ${ }^{34}$ von Bruno Meissner 1925 gewissermaßen kanonisiert worden: Er eröffnet sein Schlußkapitel über die "Ethik und Moral" mit einem Zitat aus Klio 199, um den "niedrigen Standpunkt der Babylonier und Assyrer in bezug auf die geschlechtliche Moral" zu belegen, korrigiert Herodot aber dann dahingehend, daß es sich um Hierodulen, nicht die "Weiber des Landes" handele, ${ }^{35}$ d.h. daß Klio 199 ein Reflex von kultischer Prostitution ${ }^{36}$ sei. Fünfzig Jahre nach Meissner findet sich eine sachlich identische Aussage von H. W. F. Saggs im Reallexikon der Assyriologie. ${ }^{37}$

${ }^{29} \mathrm{G}$. Rawlinson, The History of Herodotus. A New English Version, edited with copious notes and appendices, illustrating the history and geography of Herodotus, from the most recent sources of information; and embodying the chief results, historical and ethnographical, which have been obtained in the progress of cuneiform and hieroglyphical discovery, assisted by Sir Henry Rawlinson, and Sir J. G. Wilkinson, I-IV, London 1858.

${ }^{30}$ RE Suppl. 2, 252.

${ }^{31}$ A. H. Sayce, The Ancient Empires of the East. Herodotos I-III, London 1883.

${ }^{32}$ Sayce, 1.c. 115 n. 2.

${ }^{33}$ F. Delitzsch, "Zu Herodots babylonischen Nachrichten," Festschrift Eduard Sachau, ed. G. Weil, Berlin 1915, 87-102.

${ }^{34}$ B. Meissner, Babylonien und Assyrien I-II, Heidelberg 1920, 1925.

${ }^{35}$ Meissner, l.c. II 435.

${ }^{36}$ Eine Übersicht über kultische Prostitution im Nahen Osten und in Griechenland liefert E. M. Yamauchi, "Cultic Prostitution," in Orient and Occident, Fs. Cyrus H. Gordon (AOAT 22), Kevelaer/Neukirchen-Vluyn 1973, 213-22. W. Kornfeld, DBS 8, 1972, 1355-74 mit reicher Bibliographie; cf. auch U. Winter, Frau und Göttin, Freiburg/Schweiz und Göttingen 1983, insbesondere 341.

${ }^{37}$ RIA 4, 1972-75, 331-33. 
Vor allem in der klassisch-philologischen Herodot-Interpretation tradieren sich dagegen auch andere Auffassungen, die an der allgemeinen Prostitutionspflicht festhalten und sie als Opfer oder als Initiation deuten. ${ }^{38}$ Auch W. Baumgartner sieht in einer umfassenden Untersuchung der Herodoteischen Berichte über den Alten Orient ${ }^{38 a}$ zwar den Widerspruch zwischen dem Klio 199 behaupteten Brauch und "allem, was wir über babylonische Eheverhältnisse wissen", weist aber auf die Übereinstimmung der Berichte Herodots und Strabos mit dem Jeremia-Brief in dem Detail der um den Kopf gebundenen Schnur hin, deren Zerreißung eine Befreiung bedeute, und hält demnach eine Interpretation der angeblichen Sitte als Initiationsritus für möglich. In neuerer Zeit hat W. G. Lambert der assyriologischen communis opinio widersprochen und einen Zusammenhang zwischen dem vermeintlichen frühdynastischen ius primae noctis ${ }^{39}$ und Klio 199 hergestellt, der den Bericht Herodots als späten Beleg für eine ursprüngliche Defloration in Wahrnehmung des droit de seigneur erscheinen läßt. ${ }^{40}$ Auch W. Helck faßt Klio 199 als wahrheitsgemäßen Bericht auf und verbindet ihn mit antiken Nachrichten über ähnliche Bräuche im syrischen Raum, interpretiert diese aber als ritualisierte Relikte urtümlicher freier Partnerwahl durch die Frau. ${ }^{41}$

Unbeeindruckt von der historisch-kritischen Auseinandersetzung mit Klio 199 zeigen sich bis heute manche nicht-philologische Humanwissenschaften. Hier gilt Herodots Autorität noch unbedingt. In modernen Nachschlagewerken findet sich demgemäß der Klio 199 dargestellte Sachverhalt unter dem Stichwort "Prostitution" oft als unbestrittene Tatsache. Auch die Krone der Enzyklopädien macht hier keine Ausnahme. ${ }^{42}$

${ }^{38}$ Z.B. H. Stein, Herodotos, $1901^{6}, 226$ n. 16; Ph.-E. Legrand, Hérodote, Paris 1932, 192 n. 1 ("sacrifice de sa virginité"). Eine vorsichtige Vermittlung sucht O. E. Ravn, Herodotus' Description of Babylon, København 1942, 89: "Although perhaps untrue that religious prostitution was a duty 'for every native woman once during her lifetime', yet Herodotus has hardly exaggerated the part played by prostitution in the Babylonian community." Und er merkt an: Wenn der Bericht jedoch auf Wahrheit beruhe, sei er als Opfer zu deuten. Cf. schon C. G. Heyne, "De Babyloniorum instituto religioso ut mulieres ad Veneris templum prostarent, ad Herodot I 199," in Comm. Soc. Gott. Cl. Hist. et Phil. 16, 1804, 30-42. Zu anderen Deutungen (Reste von Gemeinschaftsehe, Phallusverehrung, Pubertătsritus) cf. die von W. W. How und J. Wells, A Commentary on Herodotus, Vol. I, Oxford $1961^{6}, 151$ genannte Literatur.

38aw. Baumgartner, "Herodots babylonische und assyrische Nachrichten," ArOr 18/1-2, 1950, 69106.

${ }^{39} \mathrm{Zu}$ diesem cf. zuletzt W. von Soden, ZA 71, 1981, 103-6.

${ }^{40}$ W. G. Lambert, JEOL 15, 1957-58, 196. So auch I. Seibert, Die Frau im Alten Orient, Leipzig $1973,39$.

${ }^{41}$ W. Helck, Betrachtungen zur großen Göttin und den ihr verbundenen Gottheiten, München 1971,236 sq.

${ }^{42}$ Enc. Brit., Macr. 15, $1980^{15}, 76 \mathrm{~b}$ ("An example of the former (sc. prostitution as a transitory rite) is the 'Myletta' rite of ancient Babylonia, wherein every female was required to sit in the tem- 
Innerhalb der Assyriologie nahm die Diskussion um Klio 199 im Jahre 1972 eine neue Wendung mit dem bereits erwähnten Vortrag von D. Arnaud, ${ }^{43}$ in dem die Existenz einer "prostitution sacrée" als einer vom Tempel organisierten und in seinem Interesse betriebenen Institution für keine Epoche der altorientalischen Geschichte anerkannt wird. Der Hintergrund des Zeugnisses Herodots sei der Widerspruch zwischen der literarischen Tradition über im 3. Jahrtausend existierende hochrangige Priesterinnen (n u-gig, istaritu, qadistu) und der in der Folgezeit stattfindenden semantischen Degradation ihrer Titel zu Bezeichnungen von Frauen niederen Sozialprestiges, die als Ammen tätig sind, dabei unverheiratet und den Prostituierten nahestehend ("de mœurs peu stricte"), ähnlich den kezrētu-Frauen, die niedere Dienste für den Tempel verrichten und außerdem der Prostitution nachgehen. Es handele sich hierbei aber um eine Prostitution, die vom Tempel nur toleriert, nicht aber organisiert sei. Der Widerspruch zwischen einer Tradition über hochgestellte Priesterinnen und der sozialen Wirklichkeit der mit den Titeln dieser einstmaligen Priesterinnen bezeichneten Frauen hätten die babylonischen Gebildeten durch die Erfindung der sakralen Prostitution ("en inventant 'la prostitution sacrée") zu lösen versucht.

In ihrer monumentalen Dissertation über die assyrischen Tempel schließt B. Menzel sich Arnauds Auffassung an und stützt sie mit Argumenten gegen die Existenz von Prostitution im neuassyrischen Kult. ${ }^{44}$ Sie formuliert kategorisch: "“Tempelprostitution' ist aus keilschriftlichen (sumerischen, babylonischen, assyrischen, hethitischen) Quellen nicht nachweisbar. Herodot (Hist. I 199) darf nicht als Beleg für Tempelprostitution herangezogen werden. Auch aus der Umwelt Assyriens sind dafür keine kontemporären Zeugnisse überliefert". ${ }^{45}$ Die Fluchformel der Inschrift des Kapara auf der monumentalen Statue einer Göttin vom Tell Ḥalāf, die als Strafbestimmung die Verbrennung von Söhnen für den Wettergott und die Erniedrigung von Töchtern als Prostituierte für Ištar ${ }^{46}$ enthält, wird von Menzel einerseits als Indiz für das negative Ansehen der harimtu in neuassyrischer Zeit, andererseits als möglicher Hinweis auf die Existenz von Tempelprostitution in dem nicht-assyrischen

ple of the goddess Ishtar and accept coitus from the first male who threw a silver coin in her lap.") Der Artikel nimmt hier implizit Bezug auf das einflußreiche Buch von A. van Gennep, Les rites de passage, 1909 (Paris 198133).

${ }^{43}$ Cf. n. 19.

${ }^{44}$ B. Menzel, Assyrische Tempel (Studia Pohl S.M. 10/I-II), Roma 1981, I 28, II 27* n. 306.

${ }^{45}$ B. Menzel, 1.c. II $27^{*}$ sq. n. 308.

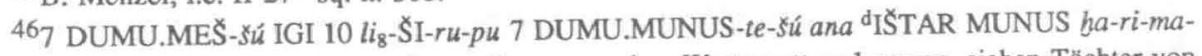
tú lu-ra-mi "Sieben Söhne von ihm soll man vor dem Wettergott verbrennen, sieben Töchter von ihm soll er der Istar als Prostituierte überlassen." Cf. B. Meissner, "Die Keilschrifttexte auf den steinernen Orthostaten und Statuen aus dem Tell Halâf," in Aus fünf Jahrtausenden morgenländischer Kultur, Fs. Max Freiherr von Oppenheim (AfO Beih. 1), Berlin 1933, 73, "Riesengöttin," Abklatsch Nr. 8:5-7; cf. 75, Abklatsche Nr. 60+61+59:5. 
Kulturkreis gewertet, dem die Statue entstammt. ${ }^{47}$ Sie vermutet daher hinter den logographisch geschriebenen Götternamen fremde Gottheiten und ist geneigt, auch in den Pönalklauseln neuassyrischer Urkunden, die eine Überantwortung von Angehörigen des Vertragsbrüchigen an Adad und/oder Ištar vorschreiben, fremden Einfluß anzunehmen. ${ }^{48}$ Die Schlüssigkeit dieser Argumentation wird allerdings nun durch das unten publizierte Tontafelfragment in Frage gestellt, aus dem unzweifelhaft Prostitution für die Göttin Ištar-Šawuška im Raum östlich von Assyrien bereits um 1400 v.Chr. nachgewiesen werden kann.

Eine forschungsgeschichtliche Ironie will, daß in demselben Jahr, in dem Arnaud die Tempelprostitution für einen historiographischen Mythos erklärte, neue Daten bekannt wurden, die für einen lokal und zeitlich eng umrissenen Kulturzusammenhang in eine andere Richtung führten. Durch die 1972 veröffentlichte Untersuchung von J. J. Finkelstein zu den kezrētu-Frauen in Kiš nach spätaltbabylonischen Texten ${ }^{49}$ ergab sich, daß diese Personen dort mit dem Tempel verbunden sind und profitable Leistungen erbringen, über deren Art den Texten zwar nichts Genaueres zu entnehmen ist, bei denen es sich Finkelsteins Meinung nach aber wahrscheinlich nicht um professionelle Prostitution handelt, obwohl "extramarital sexual activities" nicht auszuschließen seien. Jedenfalls unterstehen die kezrētu-Frauen einem Aufseher (UGULA), der die Organisation und Einnahmenverwaltung als Pfründe (parșum) Finkelstein spricht von "prerogative or benefice"-vom Palast übertragen bekommt. Solche Pfründen konnten ihrerseits Gegenstand von Rechtsgeschäften sein.

Die sonstigen Texte aus altbabylonischer Zeit liefern auch keine direkten Hinweise auf eine Verbindung der kezertum mit Prostitution; die wenigen Belege lassen nur eine niedere Funktion im Kult erkennen, und zwar sowohl in Südbabylonien ${ }^{50}$ als auch in Mari. ${ }^{51}$ Eine Nähe der kezertu zur Prostitution ist erst durch die vielzitierten loci classici jüngerer literarischer Quellen, nämlich des Gilgames-Epos ${ }^{52}$ und des Erra-Epos, ${ }^{53}$ sowie einen Eintrag in einer Synonymenliste ${ }^{54}$ gegeben, wo kezretu zusammen mit samkatu und der unzweifelhaft auch altbabylonisch schon als Prosti-

${ }^{47}$ B. Menzel, 1.c. I 28.

${ }^{48}$ B. Menzel, 1.c. I 29 , II $28^{*}$ n. 308.

49J. J. Finkelstein, YOS 13, 1972, 10. Cf. dazu M. Birot, BiOr 31, 1974, 272; C. Wilcke, RlA 5 , $1976-80,86$.

50J. Renger, ZA 58 [NF 24], 1967, 188.

${ }^{51}$ B. F. Batto, Studies on Women at Mari, Baltimore 1974, 114-18.

${ }^{52}$ Gilg. VI 165 sq.: uptahher IStar kezrēti samhāti u harimāti "Es versammelte Ištar die $k .$, §. und Huren."

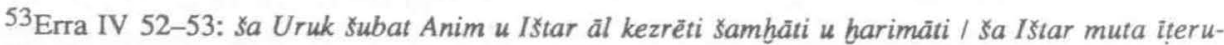
šināti-ma imnû qātułs-šn "Von Uruk, dem Wohnsitz Anus und Ištars, der Stadt der $k$., der $\xi$. und der Huren, denen Istar den Gatten nahm und die sie ihrer Verfügung zurechnete."

${ }^{54}$ Expl. malku I 82-87; cf. A. Draffkorn-Kilmer, JAOS 83, 1963, 434. 
tuierte definierten harimtu erscheint. Nur hier auch werden diese Personengruppen mit demselben sumerischen Wort kar-kid geglichen, 55 das ursprünglich nur die harimtu bezeichnet, während die kezertu in altbabylonischer Zeit mit su hur-lá geglichen ist und dies ihr Logogramm auch in späterer Zeit bleibt. Die kezertu ist allerdings nicht erst am Ende des 2. Jahrtausends in die Nähe der harimtu gerückt. Akkadische ${ }^{56}$ und hethitische ${ }^{57}$ religiöse Literatur aus Hattusa belegt sie nebeneinander und mit speziellem Bezug zu der Göttin Ištar bereits in der hethitischen Großreichszeit. ${ }^{58}$ Für die altbabylonische Zeit ist eine gewisse Nähe der drei Kategorien bisher nur darin gegeben, daß Samblatu gelegentlich als Personenname begegnet und eine harimtu $u^{59}$ oder eine kezertu ${ }^{60}$ bezeichnet, doch kommt der Name auch anderweitig vor. ${ }^{61}$

Es stellte sich bald heraus, daß die Situation der kezrētu in Kiš Parallelen in anderen Städten hatte. Ein Text aus dem altbabylonischen Sippar zeigt eine ganz ähnliche Pfründenorganisation am Tempel der Göttin Anunïtum an. ${ }^{62}$ Unter den Pfründen wird auch die harimütum, also die Abstraktbildung zu harimtu "Prostituierte", genannt. ${ }^{63}$ Nach einer Mitteilung von K. van Lerberghe ist derselbe Sachverhalt auch in unpublizierten Tafeln aus dem Archiv des Oberpriesters (kalamāhhum) Ur-Utu aus Tell ed-Dēr nachzuweisen. ${ }^{64}$ Die Texte aus der Region von Sippar und die aus Tell ed-Dēr nennen im Zusammenhang mit dem parșum der harimütum und der rēdûtum ("service as camp-follower"?65) Frauen oder Männer, die von der bisherigen For-

${ }^{55} \mathrm{Cf}$. CAD K 314.

${ }^{56}$ KUB 39, 93 Vs. 5; cf. A. Goetze, JCS 18, 1964, 95 n. 19.

${ }^{57}$ Cf. H. Otten, ZA 53, 1959, 177, 181 zu Bo 181 Rs.V 2; H. G. Güterbock, JAOS 103, 1983, 155-64 zu KUB 24, 7 I 11. Cf. p. 159 die kategorische Feststellung, daß "the SALSUHUR.LÁL/ LAL in the Hittite texts is not a hierodule".

${ }^{58}$ In Hinsicht auf die Organisation der kezrētu in altbabylonischer Zeit ist es bemerkenswert, daß die harimätu in Hattusa gleichfalls einem Aufseher (GAL, UGULA) unterstanden; cf. Otten, l.c., Güterbock, 1.c.

${ }^{59}$ So in Gilg. P.

${ }^{60} \mathrm{E}$. Szlechter, TJA 112 UMM G 22:2; cf. CAD K 315 sq., wo in sehr behutsamer Argumentation auf eine an einen Tempelfunktionär zu zahlende Ablösungssumme geschlossen wird, die die kezertu im Falle ihrer Verheiratung als Ersatz für die bis dahin von ihr erbrachten Zahlungen (kezèru, kezertu) zu leisten hatte.

${ }^{61}$ Eine Ehefrau namens Sambatu ist z.B. VAS 7, 173:2 genannt.

${ }^{62}$ R. Harris, Ancient Sippar, Istanbul 1975, 332 n. 123; H. M. Kümmel, AfO 25, 1974/77, 78; M. L. Gallery, Or 49, 1980, 334 sqq.

${ }^{63} C T$ 48, 45:2-3: (Silber) sa pa-ar-și $§[a] A[n$-nu-ni-t] $u m$ ha-ri-mu-tum (und weitere Ämter); cf. M.

L. Gallery, Or $49,1980,334$.

${ }^{64}$ K. van Lerberghe, AfO Beih. 19, 1982, 280-83.

${ }^{65}$ M. L. Gallery, 1.c. 335. 
schung ${ }^{66}$ als Ausübende der entsprechenden Tätigkeiten betrachtet werden, obwohl die betreffenden Frauen verheiratet sind, und zwar in zwei Fällen gar mit einem Priester (SANGA bzw. èrib bitim). Wahrscheinlicher ist aber, daß harimütum etc. nicht den "Status" 67 der genannten Personen bezeichnet, sondern das Amt der Aufsicht über die harimātum, die genannten Frauen und Männer also eine Art Vorsteher(innen) der Prostituiertenschaft sind. Wie dem auch sei, kann doch kein Zweifel daran sein, da $\beta$ in spätaltbabylonischer Zeit bestimmte Tempel in Nordbabylonien über organisierte Gruppen von Frauen verfügten, die als harimtum einer profitablen Tätigkeit nachgingen. Es besteht kein Grund, für diesen Personenkreis die auch altbabylonisch sonst wohletablierte Bezeichnung "Prostituierte" zu modifizieren, wie dies M. G. Gallery, ${ }^{68}$ wohl unter dem Eindruck der Argumentation Finkelsteins zu den verheirateten kezrētu, getan hat.

Der Sitz im Leben dieser Texte läßt natürlich keine Aussage darüber zu, ob die Prostitution einen kultischen Aspekt hatte, etwa als ritueller Vollzug zur Garantie der mit der sexuellen Vereinigung assoziierten Werte der Fruchtbarkeit, des Gebärens des Wachstums und Gedeihens oder als Weihung zur, wenn der Ausdruck gestattet ist, imitatio deae (was jedoch ausschließliche Bindung an den Kult der Ištar und verwandter Göttinnen voraussetzte) oder auch als Opfer. Kann demnach an einer vom Tempel organisierten Prostitution in Babylonien kurz vor der Mitte des 2. Jahrtausends kein Zweifel mehr sein, so hat doch die Verwendung der Begriffe "Kultprostitution", "prostitution sacrée" damit noch keine Berechtigung.

$\mathrm{Da} B$ die Tempelprostitution auch in der auf die altbabylonische Epoche folgenden Zeit existierte, belegt ein bisher unveröffentlichtes Fragment aus Nuzi. Es gehörte zum oberen Teil einer Tafel und füllt, nach der Krümmung der Oberfläche zu urteilen, fast die ganze Breite der Tafel aus, obwohl nur der linke Rand noch teilweise erhalten ist. Die Zeilenzählung der im folgenden gebotenen Autographie geht davon aus, daß die Zeile, die die Krümmung der Vorderseite zum oberen Rand hin füllte und von der nur eine winzige Spur erhalten ist, die erste Zeile des Textes war, doch muß dies unsicher bleiben. Ebenso unsicher ist die Höhe der Tafel. Die Maße des Fragments betragen $59 \times 48 \times 29$ mm, über seinen genauen Fundort liegen keine Angaben vor. Da das Fragment trotz seines geringen Umfangs zahlreiche lexikalische Probleme aufwirft, ist eine eingehende philologische Kommentierung nötig, bevor Schlüsse hinsichtlich der Wertung des Textes im Rahmen des hier behandelten Zusammenhanges gezogen werden können.

${ }^{66}$ M. L. Gallery, 1.c., K. van Lerberghe, 1.c.

${ }^{67}$ So K. van Lerberghe, 1.c.

${ }^{68}$ M. L. Gallery, 1.c. 


\section{SMN 1670}

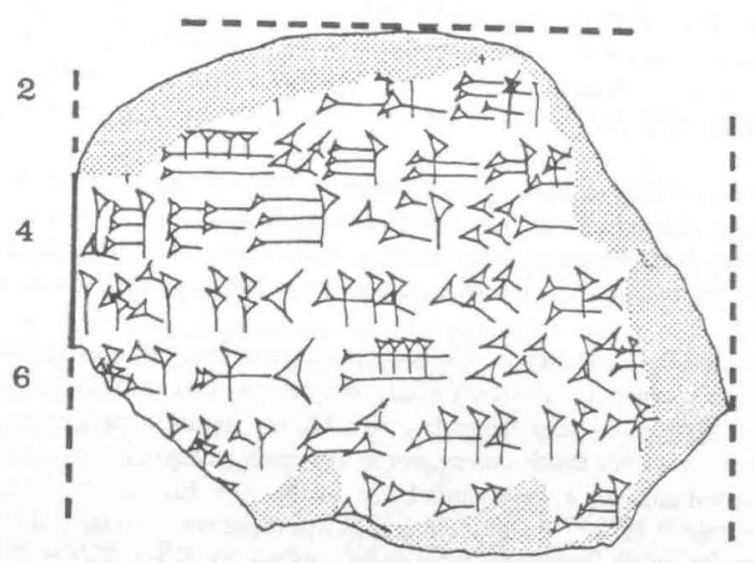
Vs. $\quad 1 \quad[\quad] \times[$
$2[\mathrm{x} \times] \times \times 5 a[$
3 r'Ú-tu-bá-al-[ti
4 ki-i-ma na-pu-[ti 0]
$5 a+n a$ ha-ri-mu-ti [0]

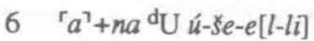
7 [uे] ' ${ }^{\top} a^{\top}+n a$ bi-ri-a-an-[na]
8 [um-t] $]$-[es]-si-i[r]-su[0]
(Rest zerstört)
Rs. (Siegelabrollung)
9 [N] $\mathrm{A}_{4} \mathrm{~m}^{\mathrm{Suúk}}$-ri-te-sup

(Siegelabrollung)
o.Rd. 10 [ ]x[
(Siegelabrollung)

11[

\section{Kommentar zu SMN 1670:}

In den ersten beiden (oder drei) Zeilen des Textes muß ein weiblicher Personenname enthalten sein, der die zur Prostitution bestimmte Frau bezeichnet. Da am Anfang der Name des Verfügungsberechtigten stehen muß, ist der Name der Frau am ehesten in 1.3 zu erwarten. Der zerstörte Anfang der Zeile reicht genau für das Determinativ aus, von dem noch eine winzige Spur vorhanden ist. Der Name ist anderweitig nicht bezeugt, doch enthält er wahrscheinlich das akkadische Personennamenelement bästi , mB bältí. Namen mit bās/lti "meine Würde" als zweitem Element sind von der altakkadi- 
schen bis zur mittelbabylonischen Zeit verbreitet, vereinzelt auch in späterer Zeit und in Assyrien, 69

In Nuzi sind solche Namen nicht sehr hăufig. JEN 452:1,5,8 und 453:10 nennt eine Frau namens Sîn-bāltī, die allerdings als hapiru bezeichnet wird, also gewiß fremder Herkunft ist und möglicherweise wie die JEN 455:2 und JAOS 55 pl. I JENu 1023:1-3 genannten hapirü aus dem "Lande Akkad" stammt. ${ }^{70}$ Auch die wenigen anderen bā̋llti-Namen in Nuzi bezeichnen Frauen mit geringem Sozialprestige: Mutu-bā̌sti JEN 501:25 ist Sklavin, Nutu-bāltī AdŠ 5:6 und Mutu-bālti Adక 8:4 ebenso. Muti/u-bāltĩ JEN 431:2,13 ist eine Witwe, die ihre Tochter zur adoptio in matrimonium servile 71 gibt.

Als erstes Glied des Namens kommt ein theophores Element oder eine Verwandtschafts- oder Herrschaftsbezeichnung in Frage. Daß Ú-tu-die syllabische Schreibung des Namens des sumerischen Sonnengottes darstellt, darf ausgeschlossen werden: Syllabische Schreibungen für Utu sind zwar vereinzelt in Nuzi nachzuweisen, doch nur in sumerischen Namen wie Ú-ta-an-ti/til ${ }^{72}$ und $U^{\prime}$-taDINGIR-GAL. 73

Da die akkadischen Personennamen von Nuzi im allgemeinen (eine genauere Überprüfung ist in Vorbereitung) keine spontanen Bildungen sind, sondern alt- und mittelbabylonischen sowie assyrischen Vorbildern folgen, es aber keine Indizien für die sumerische Lesung des meist logographisch geschriebenen Namens des Sonnengottes in akkadischen Namen gibt, ist nach einer anderen Lösung Umschau zu halten. Am wahrscheinlichsten ist die Annahme eines Fehlers für Mutu-bāltī ("der Ehemann ist meine Würde"). Es handelt sich gewiß nicht um die Darstellung von /mu/ durch

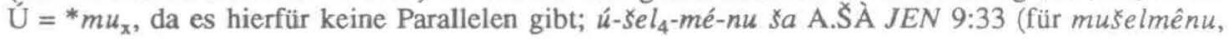
parallel zu nädinänu ša kaspî) kann hier nicht angeführt werden, da Interferenz mit der in demselben Zusammenhang hăufig gebrauchten Verbalform ư̌elmû vorliegt. Möglich wäre schließlich-was der Jubilar gesprächsweise zu bedenken gab - ein Name der Bedeutung "Ich fand meine Würde", der allerdings bereits den jüngeren $u$-Auslaut bei watû zeigen würde und ohne Parallele wäre.

1. 4: Die Ergänzung scheint die einzig sinnvolle, die Ableitung von nepû nach dem Zusammenhang die einzig mögliche. Probleme bereitet jedoch das a der ersten Silbe. Die nur aB belegte Form nipûtum "Schuldhäftling", "Pfand" ist unzweifelhaft eine pirs=ūt-Form, wie die ausnahmslose Schreibung mit dem Zeichen NI, auch in unmittelbarem Kontrast mit NE wie in CH \$116, zeigt. Das nur $\mathrm{aB}$ und vereinzelt in $\mathrm{jB}$ Königsinschriften bezeugte Verb nepûm lautet gelegentlich (frühund/oder nord-)altbabylonisch nicht um: napi $A b B$ 5, 141:17, napiat JCS 23, 33 Nr. 3:11; YOS 2, 104:21, napiā $A b B$ 1, 45:20, napâ $A b B$ 1, 93:9, inappe $T C L$ 18, 121:10, attappeam LFBD 5:11. Die Wörterbücher kennen aber auch ein aB nepitum "Frau in Schuldhaft", also das substantivierte Verbaladjektiv, für das allerdings jetzt nur noch ein einziger Beleg namhaft gemacht werden kann ( $C A D$ N/2 170b; cf. $A H w .778 b$ ). Laß sich hierzu leicht eine nicht-umlautende Form und davon abgeleitet ein Abstraktum denken, so dürfte dieses sich im gegebenen Kontext doch nicht mit kima verbinden, auf das vielmehr eine Personenbezeichnung, kein Abstraktum folgen muß. So bleibt keine Möglichkeit, napûtu als eine mit aB nipûtu semantisch gleichwertige Parallelbildung zu betrachten. Für

${ }^{69} \mathrm{Cf}$. CAD B 143b; auch in Mari ist dieser Namentyp belegt, am häufigsten als Sklavinnenname Bēlĩ-bāštī $A R M$ XVI/, 77; cf. noch Adad-bāsti $A R M$ XVI/1, 50, Abī-bāstī $A R M$ XVI/1, 46. In Tall Rimāh ist der altbabylonisch auch sonst (z.B. YOS XIII p. 58 s.v.) bezeugte Name Ilī-bāšti $O B T$ Tell Rimah 122:17 belegt.

${ }^{70} \mathrm{Cf}$. J. Bottéro, Le problème des Habiru, Paris 1954, 47, 53, 64.

${ }^{71} \mathrm{Cf}$. grundsätzlich dazu P. Koschaker, Neue keilschriftliche Rechtsurkunden aus der El-Amarna-

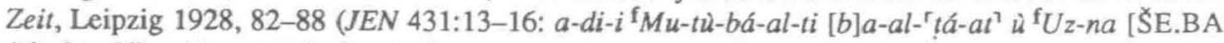
ù lu-bu-u]l!-ta [i-na-an-di-n]a-as-sit).

${ }^{72}$ Cf. NPN s.v. sowie Fs. Lacheman (SCCNH I), Winona Lake 1981, 441 (WHM 152335:4).

${ }^{73}$ Cf. NPN s.v., AAN s.v. 
andere nur in Nuzi bezeugte akkadische Nomina cf. M. Müller, WO 9, 1977/78, 30 n. 34. [Zu aB nipûtu cf. jetzt M.-F. Scouflaire, Akkadica 53, 1987, 30-34.]

1. 5: Als Abstraktbildung zu harimtu "Prostituierte" gibt es in Nuzi ähnlich wie bei anderen femininen Substantiven neben der normalen Bildung harimütu die Form harimtūtu. Für die Prostitution in Nuzi sind folgende Stellen zu verwerten:

HSS V $11^{74}$ ist die Erklärung einer offenbar seit längerem verwitweten Dame A. im Urgroßmutteralter, die ihre Tochter T. einst mit einem (wahrscheinlich zwischenzeitlich verstorbenen) Mann verheiratet hat, was A. nicht hindert, über deren (voreheliche?) Tochter E. zu verfügen. Diese letztere lebt als Prostituierte (ana harimtüti baltat), wird aber nun der wahrscheinlich verwitweten Schwiegertochter der alten Dame, M., zur Erzielung eines Brautpreises, der ihrem Unterhalt dienen soll, übergeben. Die aus dieser Verbindung zu erwartenden Urenkel werden als Nacherben für ein Feld eingesetzt, das A. ihrer Schwiegertochter M. übereignet. M. bekommt zusätzlich den Brautpreis einer weiteren Tochter oder Enkelin der A., soweit er nicht schon von dieser verbraucht ist.

Der Text zeigt, daß ein junges Mädchen vor der Ehe mit Wissen und wahrscheinlich auf Veranlassung ihrer Familie der Prostitution nachgehen und danach bei allgemeiner Kenntnis dieses Umstandes den Status einer Ehefrau erwerben konnte.

AASOR XVI 23 ist die Urkunde der adoptio in matrimonium servile sive prostitutionem, durch welche ein Mädchen von ihrem Bruder an eine hochgestellte Dame übergeben wird, die dabei das Recht erwirbt, das Mädchen wahlweise mit einem Sklaven oder einem taluhlu ${ }^{75}$ zu verheiraten oder der Prostitution nachgehen zu lassen. 76

Die Urkunde macht deutlich, daß es mit dem Status einer Dame der Oberschicht durchaus vereinbar war, Frauen ihres Personals zur Gewinnerzielung sich prostituieren zu lassen. Daß die Prostitution nicht als Schande aufgefaßt wurde, zeigt ein königlicher Erlaß, ${ }^{77}$ der es den Sklavinnen und

${ }^{74}$ Cf. E. A. Speiser, AASOR 10, 1930, Nr. 31.

${ }^{75}$ Cf. G. Wilhelm, Das Archiv des Šilwa-tešsup 2, Wiesbaden 1980, 90; M. Morrison, AfO 29/30, $1983 / 84,118$ sq.

${ }^{76}$ Der Text ist von dem Erstbearbeiter Speiser, AASOR 16, 1936 84, mißverstanden worden, wofür allerdings die unglückliche Formulierung des akkadischen Textes selbst verantwortlich ist. Die Übersetzung muß lauten: “Adoptionsurkunde der Sitanka, der Tochter des Habil-damqu. Mit ihrem Einverständnis(!) hat Hanatu, der Sohn des Habil-damqu, seine Schwester der Tulpunnaja, der Tochter des Erwe-కarri, zur Adoption gegeben, und Tulpunnaja darf Sitanka verheiraten. Wenn es Tulpunnaja gefällt, kann sie (sie) einem Sklaven geben, und wenn es ihr gefällt, kann sie (sie) einem taluhlu geben, und wenn es darüberhinaus aber der Tulpunnaja gefällt, soll Sitanka Prostitution betreiben. Und solange Tulpunnaja lebt, soll sie sie ernähren ('essen lassen'). Wenn zehn Ehemänner von ihr sterben (!-Text sg.), darf sie (sie) mit einem elften Mann verheiraten. Wenn Sitanka den Vertrag bricht und das Haus der Tulpunnaja verlăßt, muß sie zwei Minen Gold an Tulpunnaja geben, und wenn Hanatu seine Schwester Šitanka aus dem Haus der Tulpunnaja hinausführt, ist die Vereinbarung dieselbe. (Zeugen, Siegel)." [Cf. jetzt K. Grosz, SCCNH 2, 1987, 133, 137.]

${ }^{77}$ AASOR XVI 51. Eine reich kommentierte Bearbeitung findet sich in der unveröffentlichten Dissertation von M. Müller, "Die Erlässe und Instruktionen aus dem Lande Arrapba," Leipzig 1968, 7-42. Eine Kopie hat E. R. Lacheman, Sumer 32, 1976, 145 sq. vorgelegt. Der Text enthält noch einige cruces, die das Verständnis erschweren. In 1.17 (i-lu-sa-as-se) ist die von Pfeiffer und Speiser in der Erst bearbeitung vorgenommene Emendation von -lu zu qi!- ebenso wie die Lesung -tep( $C A D$ H 102a) zu verwerfen, wie schon $C A D$ E 73 und M. Müller, 1.c. 13-15, gesehen haben. Alle Versuche, die Form akkadisch zu deuten, gehen jedoch fehl. Es handelt sich um eine geläufige hurritische Verbalform, die aus dem Stamm $i l$-, dem Präteritalsuffix -oż-, dem Ergativzeichen der 3. Ps. sg. - $a$ - und dem Nominalisierungssuffix -s̄se besteht. Der Stamm e/il- ist bisher ungedeutet und 
Sklaven des Palastes (LÚ.ÌR É.GAL-li $u$ ni-i.̧ É $̧ a$ É.GAL-li) verbietet, ihre Töchter ohne Erlaubnis des Königs (bá-lu LUGAL) der Bettelei ${ }^{78}$ oder der Prostitution (harimütu) nachgehen zu lassen.

Das Appellativ harimtu ist in Nuzi mehrfach und stets in der Form harintu belegt. Eine harintu erscheint einmal in den Rationenlisten des Archivs des Silwa-teł̌̌up, ${ }^{79}$ ohne daß Genaueres über ihre Identităt zu ermitteln ist. Noch keine klare Aussage ist den an wichtigen Stellen zerstörten oder nicht hinreichend verstăndlichen Prozeßdokumentern JEN 666 und 671 zu entnehmen, die beide von demselben Schreiber ausgefertigt wurden, der auch die oben besprochene Urkunde HSS V 11 geschrieben hat. Es hat den Anschein, als sei der Anlaß der JEN 666 behandelten Klage die erbrechtlich bedeutsame Behauptung eines Mannes, seine Mutter sei die Ehefrau eines bekannten Grundbesitzers, was dieser nach der Aussage seines Bruders vor Zeugen entschieden bestreitet: fPN jâš la aššat-mi harintu kum=oz̃=au ẽpuš "PN ist mir nicht Ehefrau; ich habe (sie) als Prostituierte ... 80!" (JEN 666:13-15). In dem Protokoll einer Zeugenaussage JEN 671 erklärt der nämliche:

anderweitig selten belegt; cf. E. Laroche, $G L H 79,120$, V. Haas, $C h S$ I/1, 349 sqq. Nach dem Zusammenhang unseres Textes muß es sich um die Entsprechung zu akkadisch $§ u \overline{l u k u m}$ "gehen lassen" handeln, d.h.: $i l=o \bar{z}=a=\bar{s} \bar{s} e$ "welche er hat gehen lassen(?)". Der gesamte Satz lautet demnach: "Wer auch immer als Sklave des Palastes seine Tochter der Bettelei(?) oder Prostitution ohne (Erlaubnis des) Königs nachgehen läßt-, dessen Tochter, die er der Bettelei(?) oder Prostitution hat nachgehen lassen(?), wird man für den Palast nehmen."

78 ana ekûti. M. Müller, 1.c. 33 sqq., hat in seinem Kommentar zu AASOR XVI 51 zu Recht festgestellt, daß die Ursache des Erlasses nicht in prinzipiellen moralischen Bedenken zu suchen sei. Seine Vermutung, es gehe dem Palast um die Verhinderung eines "unkontrollierten Abfluss(es) von Arbeitskräften aus dem Palasthaushalt" (1.c. 35), geht allerdings davon aus, daß die Adressaten des Erlasses freie königliche Bedienstete, nicht Sklaven seien, eine Auffassung, gegen die Bedenken erhoben werden kőnnten, ohne daß dies an dieser Stelle ausgeführt werden soll. Ganz richtig sieht Müller aber das Motiv der angesprochenen Palastbediensteten, ihre Töchter zu prostituieren, in der Absicht, "sie ... als einträgliche zusätzliche Versorgungsquelle zu verwenden" (1.c. 35). Es wäre nicht undenkbar, daß die Intention des Erlasses in dem Interesse des Palastes liegt, über die Genehmigungspflicht einen Anteil an den Einnahmen von Töchtern seines Personals zu realisieren. Jedenfalls muß angenommen werden, daß nicht nur die Prostitution, sondern auch der als ekûtu bezeichnete Status zu Einnahmen führt, weshalb M. Müllers tentatives "Streunerin" (1.c. 12) ebensowenig befriedigen kann wie die Bedeutungsangaben der Wörterbücher (CAD E 73a: "status of homeless, destitute and unprotected girl", $A H$ w. 196a: "Stand eines Mädchens ohne Familie o.ä."). Löst man sich von der Auffassung, ekûtu sei das Abstraktum zu dem semantisch eng definierten $e k u ̄ t u$ ("homeless, destitute girl") und leitet es ebenso wie $e k \hat{t} t u$ B von $e k \hat{u}$ ab, so öffnet sich für eine mögliche

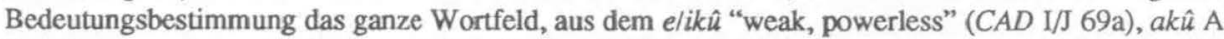
"destitute, weak, powerless, humble" (CAD A/1 283), makû A "poverty, want" (CAD M/1 140), makû "to be lacking" (CAD M/1 140), mēkûtu "lack, want" (CAD M/2 9), ukkû "darben lassen" ( $A H w$. 196a) stammen. Da die Parallelităt mit harimütu ein Status- oder Berufsabstraktum fordert, kommt als Bedeutung wohl nur "Bettelei" in Frage; cf. schon G. Wilhelm, Grundzüge der Geschichte und Kultur der Hurriter, Darmstadt 1982, 65. Eine solche Annahme muß freilich in Kauf nehmen, in Widerspruch zu der Bemerkung A. L. Oppenheims zu stehen, "the absence of references to beggars in cuneiform texts" sei "worth noting; the term pisnuqu is purely literary, very rare, and does not mean simply 'beggar"'; A. L. Oppenheim, Ancient Mesopotamia, Chicago $1977^{2}, 362$ n. 68.

${ }^{79} \mathrm{G}$. Wilhelm, Das Archiv des Šilwa-tešsup 3, Wiesbaden 1985 184, Nr. 168:10.

${ }^{80}$ Das Verb des zweiten Kolons ist auch von sprachwissenschaftlichem Interesse, da hier nicht, wie üblich, ein hurritischer Infinitiv mit einer finiten Form von akkadisch epēsu kombiniert wird, sondern eine hurritische finite Verbalform (Prät. 1. sg.) verwendet wird. 
"Seine Tochter $\mathrm{fPN}_{1}$ ist klein(?), seine Tochter $\mathrm{fPN}_{2}$ ist Prostituierte. Zur Ehefrau(?) ${ }^{81}$ hat er/habe ich(!?) (sie) nicht gemacht ... Ich habe nicht freigelassen(?? ${ }^{82}$... Mir hat sie nicht geboren(?)" 83

Das Prozeßprotokoll JEN 397 enthält die Erklärung einer Frau, derzufolge ihre Tochter Prostituierte ist. Der Text läßt die Deutung zu, daß letztere ihrem Gewerbe im elterlichen Hause nachging. ${ }^{84}$

An keiner angeführten Stelle ist ein Gesichtspunkt erkennbar, der für eine semantische Entwicklung von harimtu spricht, die von dem Sachverhalt käuflichen Geschlechtsverkehrs fortführt. Ebensowenig ist irgendwo ein Hinweis auf kultischen Bezug der Prostitution gegeben.

1. 6: ${ }^{d} U$ ist in Nuzi nicht das Logogramm für den Wettergott, der dort vielmehr stets ${ }^{d} I S ̌ K U R$ oder syllabisch geschrieben wird, sondern eine Abkürzung des Logogramms ${ }^{d} U+D A R=I క t a r{ }^{85}$ In dem Doppeltempel von Nuzi hat Istar (hurritisch Sa(w)uska) neben dem Wettergott ihr Heiligtum. Auch in anderen Stădten des Königreichs Arrapha stehen Ištar-Gestalten neben einer männlichen Gottheit an der Spitze des Pantheons und besitzen eigene Tempel. ${ }^{86}$

Der Kausativstamm von elûu "hinaufsteigen" hat zahlreiche Sonderbedeutungen. Im Zusammenhang mit Personen bewegt er sich vor allem im Zentrum des Wortfeldes: "to make a person move upward to a higher location" ${ }^{87}$ Die Spezialbedeutungen aus der Gerichts- und Militärsprache ${ }^{88}$ kommen in unserem Zusammenhang nicht in Frage. Eine Verbindung unseres Fragments mit einer bestimmten Stadt ist zwar nicht zu ermitteln, doch darf man unterstellen, daß der Tempel der Istar nicht nur in Nuzi, sondern auch an anderen Orten innerhalb der Zitadelle stand, die in aller Regel eine durch Tellbildung zumindest leicht erhöhte Position gehabt haben dürfte. Insofern wäre unser Beleg hier ohne weiteres einzuordnen. Im Zusammenhang mit dem Tempel hat $\varsigma_{u}$ lû allerdings eine Sonderbedeutung angenommen, die mit "in den Tempel hinaufbringen, dem Gott weihen" 89 und "to offer or dedicate (something) to a deity"90 - stets gesagt von Sachen, nicht von Personenwiedergegeben wird. Die Belege dafür stammen zwar überwiegend aus dem 1. Jahrtausend, aus Babylonien wie aus Assyrien, doch machen die Wörterbücher auch einige altbabylonische Stellen namhaft. $\mathrm{DaB}$ in der Tat hier eine semantische Sonderentwicklung erfolgt ist, zeigt für das $\mathrm{nA}$ die

${ }^{81}$ So nach der Kollation von H. Lewy, Or 10, 1941, 218 n. 3; cf. CAD H $10 \mathrm{lb.}$

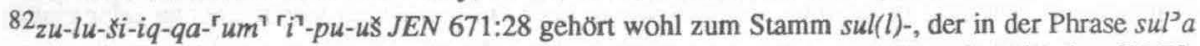
sullud- hethitisch mit arha tarna- "loslassen" wiedergegeben wird; cf. zuletzt G. Wilhelm, ZA 73,

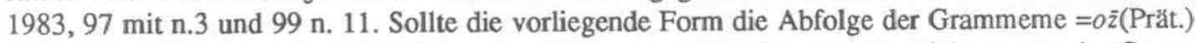
$=i($ trans. $)=k k$ (neg. $)=a u$ (Ergativ 1.Ps. sg.) enthalten, so wäre dies eine Abweichung von der Grammatik des Mittani-Briefes, die für ergativische Verbalformen in derselben Position wie hier $-k k$ - die Negation -va- vorschreibt; cf. F. W. Bush, "A Grammar of the Hurrian Language," Brandeis University $1964,200-5$.

${ }^{83}$ ia-צi ha-ni-ú-uk-ku JEN 671:29 ist gewiß zu dem Stamm han-zu stellen, der in der Ableitung ban=as̄t- mit akkadisch mära išu "einen Sohn haben" geglichen ist; cf. E. Laroche, GLH 92; cf. auch hanumašše "Fruchtbarkeit(?)" 1.c. 93. Auch diese Form widerspricht den Regeln der Grammatik des Mittani-Briefes; cf. F. W. Bush, 1.c. 202 sq.

${ }^{84} \mathrm{Cf}$. A. Fadhil, Studien zur Topographie und Prosopographie der Provinzstädte des Königreichs Arraphe (Baghdader Forschungen 6), Mainz 1983, 250 b.

${ }^{85}$ Cf. NPN 301.

${ }^{86}$ Cf. K. Deller, Or 45, 1976, 33-45; G. Wilhelm, Grundzüge der Geschichte und Kultur der Hurriter, Darmstadt 1982, 72; A. Fadhil, 1.c. 355.

${ }^{87} \mathrm{CAD}$ E $127 \mathrm{a}$.

${ }^{88} \mathrm{CAD}$ E $127 \mathrm{~b}$ sq.

${ }^{89} \mathrm{AHw}$. 209a.

${ }^{90} \mathrm{CAD} \mathrm{E} 130 \mathrm{a}$. 
Ableitung క̌̃êtútu "Tempel-Oblatin", 91 die gleichzeitig die Beschrănkung der Belege auf die Weihung von Gegenstănden relativiert. Ob elû S an der vorliegenden Stelle den Aspekt "weihen" hat oder nur auf die Richtungskomponente zielt, muB mangels weiterer Indizien unentschieden bleiben.

Anstelle des Prăsens wăre ein Perfekt oder Prăteritum zu erwarten. Fälschliche Verwendung des Präsens von $\Varangle \bar{u} l \hat{u}$ anstelle des geläufigen Perfekts und des selteneren Präteritums (z.B. HSS XIV 505:11) findet sich auch sonst: ú-se-el-li ... i-din YBC 5141:8 (cf. E. R. Lacheman / D. Owen, in Studies on the Civilization and Culture of Nuzi and the Hurrians in Honor of E. R. Lacheman, Winona Lake, 1981, 408).

11. 7-8: Die Wendung ana pirianna wussurum begegnet noch in dem Testament HSS XIX 7:19 und in der Verfügung (wohl ebenfalls Testament) HSS XIX 142:26. In allen drei Fällen ist das direkte Objekt ein weiblicher Personenname. Dies führte E. A. Speiser zu seinem von J. S. Paradise ${ }^{92}$ mitgeteilten Vorschlag, pirianna als "personal maid service" aufzufassen.93

In HSS XIX 7 verfügt der Erblasser:

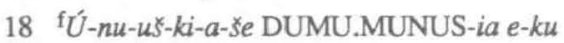

19 a-na bi-ri-a-an-na a-na ra-(ma-)ni-sá-ma

20 un-te-es-ši-ir-su ù $a-n a$ qa-ti

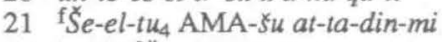

$22 a-d i-i \mathrm{f} \breve{S}_{e}-e l-t u_{4}$ bal-t tu$u_{4}$

23 ù i-pal-la-ah e-nu-ma f $\tilde{S}_{e-e l-t u_{4}}$ BA.ÚS

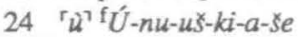

$25 a-s a r$ ha-as-bu GIN-ak ù KÙ.BABBAR.MES

26 [a-n]a qa-an-ni-sá-ma ${ }^{\mathrm{r}} r a^{\top}-k[i-i] s$

Meine Tochter, die Bettlerin(?) Unuš-kiase, lasse ich hiermit ana pirianna für sie selbst frei und übergebe (sie) hiermit ihrer Mutter Seltu. Solange Seltu lebt, wird sie (sie) ehrfürchtig behandeln. Wenn Seltu stirbt, darf Unu§-kiase gehen, wohin sie will, und das Silber (ihres Brautpreises) ist an ihren Gewandsaum gebunden.

Der Erblasser verfügt offensichtlich implizit für den Fall seines Todes das Erlöschen der $a b b u \bar{t} u$ ("văterliche Gewalt", welche insbesondere das Recht, das Mädchen zu verheiraten und den Brautpreis in Empfang zu nehmen, umfaßt), die er auch auf die Mutter seiner Tochter oder einen ihrer Brüder hătte übertragen können. Er begründet aber für seine Tochter ein andersgeartetes Abhängigkeitsverhältnis gegenüber ihrer Mutter bis zu deren Tod.

Ein vergleichbarer Sachverhalt könnte in SMN 1670 gegeben sein, wenn man diesen Text dergestalt interpretiert, daß Mutu-bāltĩ aus der abbütu entlassen wird, um in den Dienst der Göttin Ištar-Sawuska zu treten.

Ist diese Deutung richtig, bietet sich für pirianna eine Verknüpfung mit der hurritischen Wurzel *fir-an, deren Grundbedeutung etwa "Iösen" entsprechen dürfte. Von dieser Wurzel sind vor allem folgende Ableitungen bekannt: ${ }^{94}$ Die reduplizierte Bildung fir=vir=(išst)-, die hethitisch $l \bar{a}$ "lösen" entspricht, ${ }^{95}$ fir $=a d i$ "Freier, Adliger" Mit. III 26, 31, dazu fir=ad(i)=ardi "Adel" (als Per-

\section{${ }^{91} \mathrm{AHw}$. 1211a.}

92J. S. Paradise, "Nuzi Inheritance Practices," Dissertation University of Pennsylvania 1972, 119.

${ }^{93}$ Das ibid. genannte hurritische *piri "male slave" ist aus dem Eintrag ás-ta-pi-ru=ar-du u am$t[u]$ malku=`arru I 179 und sag-géme-ìr = ás-ta-pi-[ri] Ana ittišu Tafel $5 \mathrm{~A}_{3}$ 13', 8' = MSL I 73, abgeleitet; cf. E. A. Speiser, JAOS 73, 1953, 136. Das hurritische Wort für Sklave ist sonst purame; cf. Laroche, GLH 205.

${ }^{94}$ Cf. V. Haas und H. J. Thiel, Die Beschwörungsrituale der Allaiturab(b)i (AOAT 31), Kevelaer/ Neukirchen-Vluyn 1978, 245 sq.

${ }^{95}$ Cf. zuletzt G. Wilhelm, ZA 73, 1983, 97. 
sonengruppe), 96 und vielleicht pir=ang=umma "fliehen" AASOR XVl 52:18. *pirianni könnte also etwa "Freiheit", im konkreten Fall "Muntfreiheit" bedeuten. Die grammatische Bildungsweise bleibt leider dunkel.

\section{Der Text kann demnach folgendermaßen übersetzt werden:}

[...] die Mutu(!)/Ütu-bāll tĩ (meine Tochter(?))] habe ich/hat er(!) als Schuldhäftling zur Prostitution zu (der Göttin) Ištar (Šawußka) hinaufgebracht, und zur Muntfreiheit(?) habe ich/hat er sie freigelassen [...]. Siegel des Sukri-teß̌up [...].

Das Fragment zeigt damit unzweifelhaft die Existenz von Prostitution im Rahmen der Tempelorganisation an. Der juristische Charakter der Urkunde läßt eine metaphorische Deutung der Erwähnung des Gottesnamens, wie sie von B. Menzel für die oben erwähnte neuassyrische Fluchformel angenommen wurde, nicht zu. Ebensowenig kann hier die Interpretation Arnauds Anwendung finden, es handele sich nicht um eine vom Tempel organisierte, sondern nur tolerierte Prostitution, da die Prostitution ausdrücklich als Zweck (ana harimüti) der Überstellung des Mädchens in die Sphäre der Göttin Ištar-Šawuška angegeben wird. Darüberhinaus liegt ein materielles Interesse des Tempels vor, da die zukünftige Prostituierte als Personenpfand bezeichnet wird und ihre Leistung gewiß ähnlich wie die eines zur Darlehenssicherung gestellten tidennu als Zinsäquivalent betrachtet wird.

Eine genaue zeitliche Fixierung des Fragments innerhalb der fast 150 -jährigen ${ }^{97}$ Laufzeit der Nuzi-Texte ist aufgrund fehlender prosopographischer Daten nicht möglich. HSS XIX 7, das die gleiche Formulierung verwendet, gehört der Zeit der vorletzten Schreibergeneration an und fällt damit in die Mitte des 14. Jhdts.

Neben die altbabylonischen Indizien für die Existenz von Tempelprostitution tritt

${ }^{96}$ Cf. zuletzt G. Wilhelm, Or. NS 54, 1985, 491.

${ }^{97}$ Die Laufzeit der Nuzi-Texte hängt im wesentlichen von dem Ansatz der Generationenlänge ab. Zu dieser im Allgemeinen cf. M. B. Rowton, JNES 17, 1958, 100-2 und O. R. Gurney, in Anatolian Studies Presented to Hans Gustav Güterbock, Istanbul 1974, 108 sq. Die Berechnungen Rowtons ergeben 148-222 Jahre für die Regierungen von sieben Generationen, d.h. ca. 21,1 - 31,7 Jahre für eine Generation. Daß die untere Marge eher dem Normalfall nahekommt als die obere, zeigt ein Vergleich mit den osmanischen Sultanen von Mehmed I. bis Ahmed I., die in ununterbrochener Vater-Sohn-Folge 204 Jahre regierten. Als durchschnittliche Regierungszeit ergeben sich 20,4 Jahre, als durchschnittliches Alter bei der Geburt des Nachfolgers (der in 7 von 10 Fällen der älteste Sohn ist) 21,5 Jahre. Rechnen wir mit einem Generationenabstand von 23 Jahren und stellen in Rechnung, daß den fünf Generationen der Familie des Tebip-tilla sieben in der Familie des Kizzuk (cf. dazu G. Dosch und K. Deller, SCCNH 1, Winona Lake 1981, 91-113) gegenüberstehen, so ergeben sich als Laufzeit für die Nuzi-Texte 161 Jahre, die aber noch zu reduzieren sind, da für die letzte Generation vor der Zerstörung nicht die volle Länge veranschlagt werden kann. G. Dosch und K. Deller, 1.c., bezeichnen ca. 1600-1400 als den "realistischere(n) Ansatz" für die Laufzeit der NuziTexte. Auch A. Fadhil, STPPKA, Mainz 1983, 1 setzt ca. 200 Jahre für die Nuzi-Texte an, bietet aber mit "1450-1250" oder "1400-1200" unzutreffende absolute Zahlen. Aller Wahrscheinlichkeit nach fand die Zerstörung von Nuzi im Zusammenhang mit der Zerstörung des Mittani-Reiches und damit im dritten Viertel des 14. Jahrhunderts statt. [Cf. jetzt D. Stein, ZA 79 (1989) 36-60.] 
somit ein Beleg aus der 2. Hälfte des 15. oder der 1. Hälfte des 14. Jhdts., der dasselbe Phänomen in einem Gebiet bezeugt, das später zu Assyrien gehört. Die neuassyrischen Hinweise auf Prostitution im Dienste der Istar müssen auf diesem Hintergrund wohl anders bewertet werden, als B. Menzel dies tat. ${ }^{98} \mathrm{Gewiß}$ ist dies noch zu wenig, um einen Traditionsstrang zu knüpfen, der bis zu Klio 199 reicht. Als "Erfindung" allerdings kann die altorientalische Tempelprostitution nicht mehr bezeichnet werden, und die traditionelle Herleitung von Klio 199 aus einem Mißverständnis babylonischer Tempelprostitution kann sich nun erstmals auf positive Aussagen der Keilschriftquellen stützen.

${ }^{98}$ Cf. n. 47. [Unter der nach Abschluß des Manuskripts (Juni 1986) erschienenen Literatur ist vor allem W. Fauth, "Sakrale Prostitution im Vorderen Orient und im Mittelmeerraum," JbAC 31, 1988, 24-39, zu nennen (frdl. Hinweis von H. Niehr).] 